\title{
Spotlight on the Fear of Cancer Recurrence Inventory (FCRI)
}

\author{
Allan Ben Smith (iD ${ }^{1,2 *}$ \\ Daniel Costa ${ }^{2,3, *}$ \\ Jacqueline Galica (DD $^{2,4,5, *}$ \\ Sophie Lebel ${ }^{2,6, *}$ \\ Nina Tauber iD ${ }^{2,7}, *$ \\ Sanne Jasperine van Helmondt (iD) $2,8,9, *$ \\ Robert Zachariae (iD) $2,7 * *$ \\ 'Centre for Oncology Education and \\ Research Translation (CONCERT), \\ Ingham Institute for Applied Medical \\ Research \& University of New South \\ Wales, Liverpool, New South Wales, \\ Australia; ${ }^{2} \mathrm{FOR}$ wards, International \\ Psycho-Oncology Society Fear of Cancer \\ Recurrence Special Interest Group, \\ Ottawa, Canada; ${ }^{3}$ School of Psychology, \\ University of Sydney, Sydney, New South \\ Wales, Australia; ${ }^{4}$ School of Nursing, \\ Queen's University, Kingston, Ontario, \\ Canada; ${ }^{5}$ Division of Cancer Care and \\ Epidemiology, Queen's Cancer Research \\ Institute, Kingston, Ontario, Canada; \\ ${ }^{6}$ School of Psychology, University of \\ Ottawa, Ottawa, Ontario, Canada; ${ }^{7}$ Unit \\ for Psychooncology and Health \\ Psychology (EPoS), Department of \\ Oncology, Aarhus University Hospital \\ and Department of Psychology and \\ Behavioural Science, Aarhus University, \\ Aarhus, Denmark; ${ }^{8}$ Department of \\ Medical and Clinical Psychology, Tilburg \\ University, Tilburg, the Netherlands; \\ ${ }^{9}$ Scientific Research Department, Helen \\ Dowling Institute, Bilthoven, the \\ Netherlands
}

*These authors contributed equally to this work

Correspondence: Allan Ben Smith FORwards, Psycho-Oncology Research Group, Ingham Institute, Liverpool Hospital, Locked Bag 7I03, Liverpool BC NSW, BC NSW, Australia

Tel +6I 287389244

Email ben.smith@unsw.edu.au
This article was published in the following Dove Press journal: Psychology Research and Behavior Management

Abstract: Fear of cancer recurrence (FCR) is a pervasive concern for people living with cancer. The rapidly expanding FCR literature has been weakened somewhat by use of miscellaneous FCR measures of varying quality. The Fear of Cancer Recurrence Inventory (FCRI) has been widely used in observational and intervention studies and the FCRI severity subscale, also known as the FCRI-Short Form (FCRI-SF), is often used to identify potential cases of clinically significant FCR. Given the FCRI's increasing use in research and clinical practice, we aimed to provide an overview, critique, and suggested improvements of the FCRI. Studies citing the original FCRI validation paper were identified and synthesised using narrative and meta-analytic methods. The 42-item FCRI has demonstrated a reasonably robust 7-factor structure across evaluations in multiple languages, although certain subscales (eg, Coping) demonstrate suboptimal reliability. Confirmation of the cross-cultural equivalence of several FCRI translations is needed. Meta-analysis of FCRI-SF scores revealed a combined weighted mean score of 15.7/36, a little above the lowest proposed cut-off score $(\geq 13)$ for clinical FCR. Depending on the FCRISF cut-off used, between $30.0 \%$ and $53.9 \%$ of the cancer population (ie, patients and survivors) appear to experience sub-clinical or clinical FCR. Higher FCRI scores were associated with younger age and female gender, pain/physical symptoms and psychological morbidity, consistent with the FCR literature generally. Issues regarding the application and interpretation of the FCRI remain. Whether the FCRI is well suited to assessing fear of progression as well as recurrence is unclear, the meaningfulness of the FCRI total score is debatable, and the use of the FCRI-SF to screen for clinical FCR is problematic, as items do not reflect established characteristics of clinical FCR. Refinement of the FCRI is needed for it to remain a key FCR assessment tool in future research and clinical practice.

Keywords: cancer, fear of recurrence, survivorship, oncology, questionnaire, self-report measure

\section{Introduction}

Fear of cancer recurrence (FCR) is defined as the fear, worry, or concern that cancer may come back or progress. ${ }^{1}$ Managing FCR is the number one unmet need of cancer survivors post cancer treatment. ${ }^{2}$ It manifests itself on a continuum with an estimated $49 \%$ of cancer survivors reporting moderate to severe levels of FCR, ${ }^{2}$ often referred to as clinical FCR ${ }^{3}$ FCR does not appear to decrease over time, and thus if left unaddressed, it can become a lifelong concern. ${ }^{2,4,5}$ At the individual level, clinical FCR has demonstrated association with psychological distress, impaired physical, emotional, cognitive, and social functioning, and lower overall quality of life (QOL). ${ }^{3-5}$ At the system level, clinical FCR is associated with increased health-care costs. ${ }^{6-8}$ 
Research on FCR is growing rapidly, with increasing data on its prevalence, correlates and treatment across cancer types. ${ }^{2,9}$ Efforts to systematically capture FCR have been hampered by a lack of agreement regarding a gold standard measure and use of miscellaneous assessment tools of variable quality. A 2012 literature review of FCR self-report measures found 20 multi-item scales, ${ }^{10}$ with a 2013 updated review identifying 12 additional scales. ${ }^{2}$ Many of these measures provide little or no psychometric data and/or have been used in few studies, limiting external validity. Furthermore, several are cancer site-specific and available in only one language limiting comparisons across studies. Finally, few instruments provide a clinical cut-off score to identify those potentially needing intervention. ${ }^{10}$

The Fear of Cancer Recurrence Inventory (FCRI) ${ }^{11}$ is a 42-item scale that is recognized as one of the psychometrically strongest measures of FCR and is recommended for research and clinical purposes when the context warrants in-depth assessment of FCR. ${ }^{10}$ The FCRI was developed by a panel of six psycho-oncology experts in the early 2000s. ${ }^{11}$ They used the following definition of FCR to develop the initial pool of items: the fear or worry of the possibility that the cancer will return or progress in the same organ or in another part of the body. ${ }^{11,12}$ They generated items $(n=75)$ based on a literature review, a cognitive-behavioral conceptualization of FCR, and Diagnostic and Statistical Manual of Mental Disorders (DSM) diagnostic criteria for various anxiety and somatoform disorders. This prototype was successfully pilot-tested for clarity and appropriateness with 10 cancer survivors. It was intended to be a selfreport measure with a one-month response time-frame.

Clinical observations that FCR may differ between patients (eg, those who seek excessive medical attention vs those who use avoidance strategies) ${ }^{13}$ led the FCRI developers to a multidimensional conceptualization of FCR. They postulated that FCR encompasses factors including potential triggers activating FCR, the severity of intrusive thoughts associated with FCR, psychological distress and functioning impairments related to FCR, selfawareness regarding the intensity of FCR, and various coping strategies that can influence FCR. ${ }^{11}$ According to the developers, it was important to assess components such as intrusive thoughts and functioning impairments associated with FCR to better identify patients experiencing dysfunctional FCR who may require professional intervention.
The original version was developed in FrenchCanadian and validated in a sample of 600 breast, colon, prostate and lung cancer survivors $35 \%$ response rate to a mailout survey), but was designed for use with all cancer patients. ${ }^{11}$ It demonstrated excellent internal consistency, one-month test-retest reliability, and face, content, and construct validity (see details below). A factor analysis of the FCRI resulted in 42 items evaluating seven FCR components (Triggers, Severity, Psychological Distress, Functioning Impairment, Insight, Reassurance, and Coping Strategies). The FCRI was forward-backward translated into English, which was subsequently empirically validated and demonstrated similar psychometric properties to the original French version (details also below). ${ }^{14}$

The FCRI has a short-form (its Severity subscale, also referred to as the FCRI-SF) with an empirically validated cut-off score distinguishing "normal" from "clinical or pathological" FCR. ${ }^{3}$ Given the FCRI's increasing use in research and the need for reliable and valid instruments that could be used to screen for clinical FCR in clinical practice, our objectives were to:

1. Summarize evidence regarding the factor structure and psychometric properties of the FCRI;

2. Synthesize evidence regarding the prevalence, severity and correlates of FCR according to the FCRI;

3. Discuss limitations and potential improvements of the FCRI.

\section{Methods}

This state-of-the-art review ${ }^{15}$ comprised three components. Authors primarily responsible for each component are shown in brackets.

1. A narrative synthesis of peer-reviewed literature regarding the FCRI factor structure and psychometrics and identified correlates of FCR (JG, SvH);

2. A meta-analysis of the prevalence and severity of FCR according to the FCRI (NMT, RZ);

3. Discussion of FCRI limitations and potential improvements (DC, SL, NMT, BS, RZ).

The Scopus database was used to identify peer-reviewed articles published in English citing the original FCRI validation paper, ${ }^{11}$ from its publication date in March 2009 to March 2020. No additional searches of any other 
information source were conducted. All article types were eligible for inclusion in our narrative literature review, which involved grouping articles together according to review objectives, tabulating relevant results and evaluating patterns of results. Article quality was not assessed.

As a supplement to the narrative synthesis of FCRI studies, a meta-analysis of the severity and prevalence of FCR was conducted. Studies for inclusion were peerreviewed articles in English citing the original FCRI validation paper ${ }^{11}$ that reported mean FCRI total scores, and/ or mean FCRI-SF scores, and/or percentages using the FCRI-SF cut-offs of $\geq 13$, $\geq 16$, or $\geq 22$. One author (NMT) screened all relevant articles for inclusion in the meta-analysis and extracted the data. A second author (RZ) double-checked $30 \%$ of the included reports and the extracted data. Studies were coded according to the following characteristics: Cancer type, patient type (survivors [no active cancer present] vs patients [active cancer present]), country where the study was conducted, average time since diagnosis, study type [observational vs trial] and sample size.

To produce an overall estimate of FCR severity and prevalence according to the FCRI, we conducted a number of meta-analyses, calculating with random effects models the combined inverse variance weighted mean scores and prevalences of FCR for both samples of patients in active treatment for their cancer and samples consisting mostly/ entirely of cancer survivors who had completed their primary treatment. If results were heterogeneous, ie, if the variance of mean FCRI scores and prevalences were likely to reflect true between-study differences beyond random error as indicated by an $I^{2}$ statistic $>0.0$, we explored possible between-study differences with subgroup analyses, meta-ANOVAs, and meta-regression.

The discussion of FCRI limitations and potential improvements was informed by the empirical findings of this review, limitations noted in included studies, and the authors' experiences of using the FCRI.

\section{Results and Discussion}

At the time of search, 198 articles citing the original FCRI validation paper were identified. Studies reporting quantitative data from the FCRI were identified for inclusion in the meta-analytic component of the review. Eighteen studies were included in the narrative synthesis of FCRI factor structure and psychometric properties findings. Forty-six studies were included in the meta-analysis of FCR prevalence and severity according to the FCRI. All other studies were considered for inclusion in the literature review component.

\section{FCRI Factor Structure and Psychometric Properties Cross-Cultural Validity}

To date, the FCRI has been translated from French into Chinese, ${ }^{16}$ Danish, ${ }^{17}$ Dutch, ${ }^{18}$ English, ${ }^{14}$ Korean, ${ }^{19}$ Mandarin, ${ }^{20}$ Persian, ${ }^{21}$ and Turkish, ${ }^{22}$ all using forwardsbackwards translation. However, testing cross-cultural equivalence with a sample of bilingual cancer patients has received little attention, perhaps because using bilingual samples may not be achievable for all translations and countries. Cross-cultural equivalence has been established for two FCRI translations: the English translation, by means of a repeated-measures analysis of variance (in 42 cancer patients), ${ }^{14}$ and the Korean translation, by means of ranking similarity of FCRI items on a 7-point Likert scale (in 6 cancer patients, 8 nurses, and 18 physicians). ${ }^{19}$ Responses to FCRI items were not influenced by language nor order of administration. ${ }^{14,19}$ Three studies reported problems with composing bilingual cancer patients samples for cross-cultural validation, in two studies nurses, physicians, and an expert panel were used. ${ }^{17,19,21}$

\section{Factor Structure}

The factor structure of the different FCRI translations has been examined using confirmatory factor analysis (CFA). Comparison of the studies is hampered by the different CFA approaches used and the different types and numbers of fit indices reported. ${ }^{23}$ In some studies, only the model fit of the 7-factor model was reported, while in others, an improved model fit was also reported (depending on the estimation method and CFA software used). Moreover, two studies reported model fit of alternative models. ${ }^{22,23}$ One study compared the factor structure results of all FCRI translations and found that when the same cut-off criteria were applied to the studies' fit indices, quite different results were achieved. ${ }^{23}$ Furthermore, results varied between and within translations. ${ }^{23}$ For example, two CFA studies of the same English translation reported a strong and a weaker model fit. ${ }^{14,24}$ Thus, differences in results cannot be attributed to translations or cultural differences only. $^{23}$

Two studies aimed to improve the FCRI by conducting exploratory factor analyses (EFA). Eyrenci et al (2018) found a 5-factor solution in a Turkish sample, which gives slightly better results than the Turkish version of 
the original 7-factor FCRI. ${ }^{22}$ Van Helmondt et al (2020) found a 4-factor solution in a Dutch sample, ${ }^{23}$ however, subsequent CFA resulted in a poor model fit of this new 4-factor model and an acceptable model fit of the original 7-factor model to data from two new samples. ${ }^{23}$ Collectively, FCRI factor analysis results are acceptable and fairly consistent across translations, but there is potential for improvement of the 7-factor FCRI.

\section{Reliability}

The internal consistency of the 7-subscale FCRI has been explored in samples of Dutch, ${ }^{18,23}$ English, ${ }^{14}$ French, ${ }^{11,13}$ Korean, ${ }^{19}$ and Mandarin $^{20}$ speaking participants. Generally, the FCRI and its subscales demonstrate good to excellent internal consistency; however, when using Ponterotto and Charter's matrix ${ }^{25}$ for estimating the adequacy of internal consistency coefficients, some reliabilities are categorized as less than ideal. According to Ponterotto and Charter's guidelines, ${ }^{25}$ the internal consistencies of the Reassurance and Coping Strategies subscales in several studies ${ }^{11,13,14,18,19,23,24,26}$ can be categorized as fair or moderate, which corroborates with other empirical analyses ${ }^{22,27}$ suggesting problematic features of these subscales.

\section{Test-Retest Reliability}

The test-retest reliability of the FCRI has been examined across languages using reported intervals between $14^{21}$ and 206 days. ${ }^{19}$ Although no studies have compared the test-retest reliability of the FCRI over time, plotting the reliabilities from available cross-sectional studies suggests that there is minimal variation (eg, 0.96 and 0.84 for 14day and 16-day intervals, respectively, to 0.90 at 206 days). According to guidance on reliability and validity of patient-reported outcome measures,${ }^{28}$ the overall FCRI demonstrates good to excellent reliability, with a weighted, pooled test-retest correlation of $0.89 \quad(95 \%$ CI: 0.80-0.98). ${ }^{11,17,19}$ FCRI subscales generally demonstrate acceptable reliability, with the Reassurance ${ }^{14,18}$ and Coping Strategies ${ }^{18,19}$ subscales most often having suboptimal reliability.

\section{Validity}

The convergent, concurrent criterion, divergent and discriminant validity of the FCRI have been explored using various measures with diverse cancer samples. The convergent validity of FCR measures (eg, Fear of Recurrence Questionnaire ${ }^{11,20}$ ) or measures of more generalised anxiety (eg Penn State Worry Questionnaire ${ }^{17}$ ) are often examined in relation to the FCRI, with results demonstrating a convergence of constructs being measured. Similarly, the FCRI's divergent validity is most often determined using QOL measures (eg, EORTC QLQ-C30 ${ }^{14,29}$ ), whereas concurrent criterion validity has often been determined using the Impact of Events Scale ${ }^{11,22,29}$ or Hospital Anxiety Depression Scale, ${ }^{11,19-21,29}$ collectively indicating that the FCRI assesses a conceptually distinct construct from these measures.

\section{FCR Severity and Prevalence According to the FCRI}

A comprehensive 2013 systematic review of the literature found that $22 \%$ to $87 \%$ of cancer survivors reported moderate to high levels of FCR, and that high levels were experienced by between $0 \%$ and up to $15 \%$. $^{2}$ These estimates were based on several heterogeneous FCR measures, and only two studies used the FCRI. ${ }^{11,13}$ However, a growing number of studies now use the FCRI, making it easier to compare FCR severity and prevalence across cancer diagnoses, sociodemographic groups, and cultures, and enabling comparisons of FCR intervention trial results. We identified studies of 46 independent samples with published data on FCR assessed with either the full FCRI or the FCRI-SF obtained from a total of 14,092 cancer patients and survivors. Eleven studies were conducted with Australian, 10 with Canadian, 5 with Dutch, and 3 with US samples, with the remaining studies conducted in various European and Asian countries. In these studies, data were generally presented as mean FCRI-total scores, and/or mean FCRI-SF scores, and/or as percentages using the FCRI-SF cut-offs of $\geq 13,6,7,30-32,33-36$ $\geq 16^{17,33,37,38}$ or $\geq 22 .{ }^{36,37}$ See Table 1 for further details.

FCRI-total scores (score range 0-168) were reported in 25 studies, with mean scores ranging from 39.8 in a sample of prostate cancer survivors ${ }^{11}$ to 113.5 in a sample of gynecological cancer survivors. ${ }^{39}$ The reported standard deviations ranged from 18.6 to 39.5 with an average of 28.2, samples sizes varied from 27 to 1984 and the mean time since diagnosis ranged from 4.1 months to 16.5 years. As shown in Table 1, the overall combined, weighted mean FCRI-Total score across all cancer types was 65.2 (95\% CI: 58.0-72.3).

FCR severity, assessed with the FCRI-SF (score range 0-36), also varied considerably across cancer patients and survivors. In the 33 studies which provided data on FCRISF, mean scores ranged from 10.4 in a sample of 


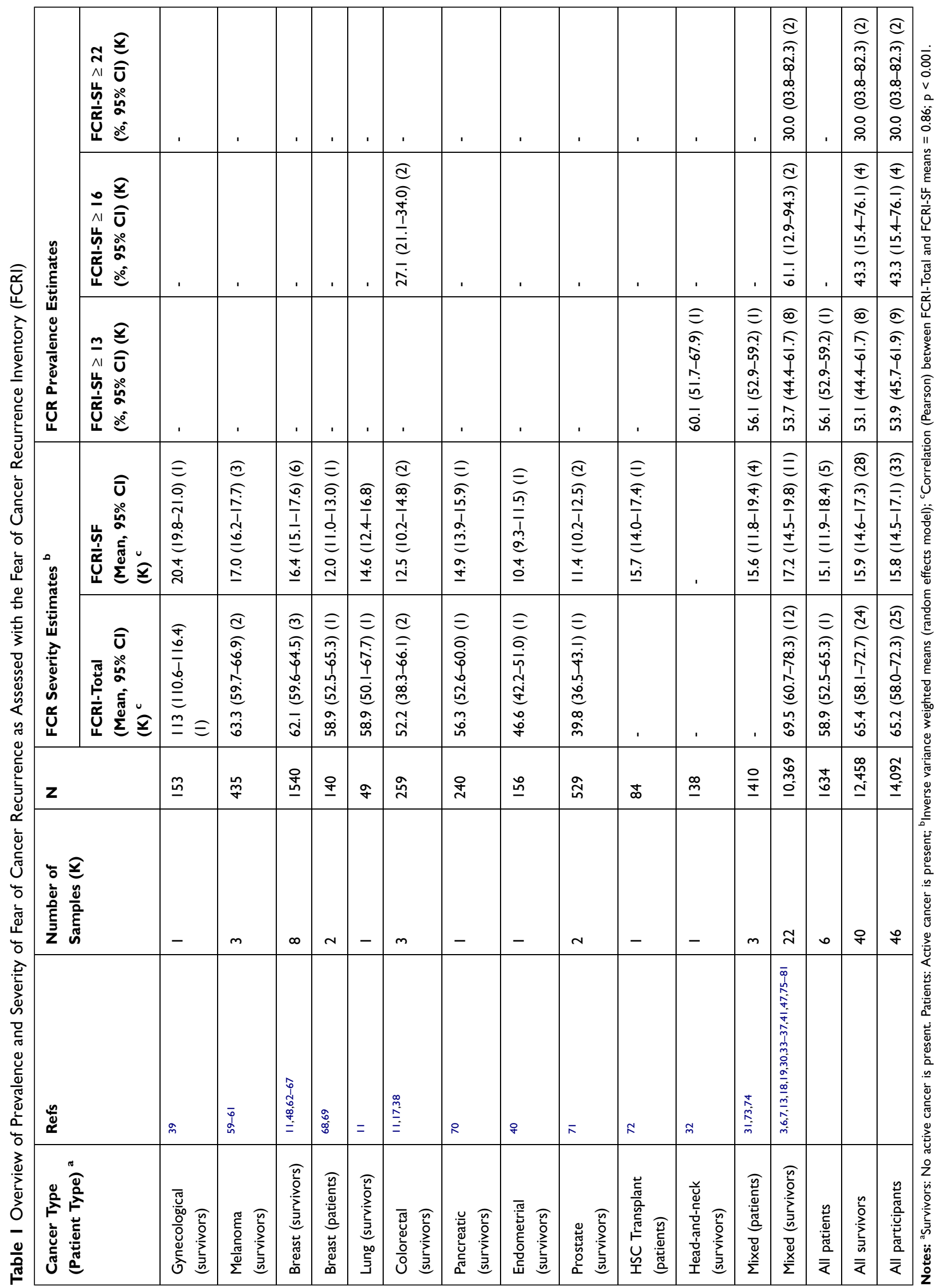


endometrial cancer survivors ${ }^{40}$ to 20.4 in a sample of gynecological cancer survivors. ${ }^{41}$ Standard deviations ranged from 3.4 to 8.3 with an average of 6.5. Sample sizes ranged from $38^{42}$ to $1984^{13}$ and mean time since diagnosis varied from 2.2 months $^{42}$ to 8.9 years. $^{30}$ As seen in Table 1, the aggregated, weighted mean FCRI-SF score was 15.8 (95\% CI: 14.5-17.1), which is above the lowest proposed cut-off $(\geq 13)$ for clinical FCR.

The largest number of studies investigated samples of patients and survivors with mixed cancers, with mean FCRI-SF scores close to or above the $\geq 16$ cut-off score for clinical FCR. In the few studies of patients with a specific cancer type, the highest levels of FCR were observed in gynecological, melanoma, and breast cancer survivors and the lowest levels in prostate cancer survivors, but given that few studies have explored FCR in samples of specific cancer types, these results should be interpreted cautiously. Cancer patients in active treatment tended to have slightly lower FCRI-SF scores (mean = 15.1) than survivors (mean $=15.9)$, but the difference did not reach statistical significance $(\mathrm{Q}=0.19, p=0.67)$.

A surprisingly small proportion of studies reported the percentages of patients with scores taken to indicate clinical FCR. As seen in Table 1, in the nine studies $^{6,7,30-32,33-36}$ using $\geq 13$ on the FCRI-SF as a cutoff, more than half $(53.9 \%)$ of participants scored above the cut-off. In the four studies ${ }^{17,33,37,38}$ with data on the $\geq 16$ cut-off, approximately $43.3 \%$ had scores indicating clinical levels of FCR. In the two samples ${ }^{36,37}$ with data on the $\geq 22$ cut-off, approximately one third ( $30 \%)$ had scores suggesting clinical levels of FCR.

Taken together, the available data suggest that FCR is a pervasive and prevalent problem across cancer diagnoses, with - depending on the chosen cut-off - between a quarter and half of the cancer population being likely to experience sub-clinical or clinical levels of FCR. The results should, however, be interpreted in the light of a number of methodological limitations. First, several of the studies reporting data on FCR severity are clinical trials where the participants may differ from the general cancer population, given that samples in clinical trials are often based on specific inclusion criteria. However, when we compared mean FCRI-SF-scores from 22 observational studies (mean $=15.6)$ with those obtained from ten clinical trials (mean $=15.8)$ no differences were found $(\mathrm{Q}=0.01$; $p=0.94)$. Second, most studies only reported sample means and failed to explore differences between subgroups or changes over time. So far, only one study of breast and colorectal cancer survivors ${ }^{42}$ has explored FCR trajectories based on the commonly used FCRI-SF cut-offs. Three trajectories were identified: $62.4 \%$ remained lowstable (mean range 5.82-7.81), 29\% experienced persistent high levels (mean range 15.12-17.48), and 8.3\% experienced initial high levels $(\geq 22)$ but recovered over time. Further trajectory analyses may clarify the extent to which certain subgroups of cancer patients and survivors may be particularly impacted by high FCR.

Meta-regression was used to examine associations between the region (Australia and New Zealand, Europe, North America, Asia) and language (English, Not English) of FCRI administration, time since diagnosis, and FCRI scores. No differences in FCRI-total scores were found between regions $(\mathrm{p}=0.17-0.75)$ or language $(\mathrm{p}=0.62)$ of administration. However, FCRISF scores were significantly higher in English (18.0; 95\% CI: 16.0-20.0) than non-English language samples (14.3; 95\% CI: 12.9-15.7; Beta=3.7; $\mathrm{p}=0.003$ ). The difference remained statistically significant (Beta $=4.1$; $\mathrm{p}=0.014$ ) when adjusting for cancer type, patients in active treatment vs cancer survivors, and region. The reason for this difference is unclear, but it may be that the greater prevalence of mindfulness and acceptancebased cultural beliefs/practices in some non-English speaking countries reduces the impact of FCR. Further research to establish the cross-cultural equivalence of FCRI translations is needed to ensure that differences in FCRI-SF scores according to language of administration are not an artefact of translation.

In the 21 studies with data on both FCRI-total scores and time since diagnosis, a statistically significant inverse association was found (Beta $=-0.14, p=0.046$ ). In contrast, a positive association was found between FCR severity and time since diagnosis in the 25 studies reporting FCRI-SF scores (Beta $=0.05, \mathrm{p}=0.049$ ), possibly suggesting that, in contrast to previous claims that FCR is stable over time, it may in fact worsen. However, several caveats should be noted regarding this finding: 1) It is a weak association; 2) Generally, only very rough estimates of time since diagnosis were available; 3) FCRI-SF scores for longer-term survivors may be inflated by an item assessing the duration of thoughts about recurrence (See below). To further explore possible moderating factors and to establish the more precise estimates of prevalence of FCR, individual participant data meta-analyses are required. 


\section{FCR Correlates According to the FCRI}

The FCRI has been used to evaluate various demographic, clinical, and psychosocial correlates of FCR. Variables commonly associated with higher FCR in these studies include younger age; $;^{30,36,43,44}$ female gender; ${ }^{36,44,45}$ pain; ${ }^{45,46}$ poor sleep ${ }^{37}$ or fatigue; ${ }^{45}$ and higher anxiety and depression. ${ }^{43,47,48}$ There is some indication that the levels of FCR may vary by cancer diagnosis; ${ }^{30,45}$ however, further clarification is necessary as no systematic comparisons taking other between-sample differences into consideration have been conducted. Similarly, time since diagnosis/treatment has been explored in relation to FCR level, ${ }^{31,43,44,48}$ with results varying between studies and the measure (FCRI total or FCRI-SF) used, as observed above.

\section{FCRI Limitations and Future Directions Debate Over Using FCRI-Total Score versus Severity Score as an Outcome}

In addition to the subscale scores representing seven domains related to FCR, the total score, ie, an aggregate of all 42 items, is also commonly used in research. This practice is typically justified by invoking the high internal consistency of the full item set. ${ }^{11}$ Although a single score is clearly simpler to analyse and report, the interpretation of this total score is questionable. Some of the present authors ${ }^{49,50}$ have argued that this aggregate combines level of fear with some of its antecedents and consequences, ignoring possible causal structure among the domains and obscuring the possibility that different respondents can arrive at similar, even identical, total scores via very different profiles of subscale scores.

Meaningful interpretation of the total score requires not only empirical validation but conceptual clarification regarding what construct it assesses. The total score certainly captures far more than FCR, as it is commonly defined, ${ }^{1}$ and even if data are able to support a higher-order factor capturing shared variance in the seven subscales, it is unclear what this higher order factor represents. Regarding empirical validation, a high Cronbach's alpha does not support unidimensionality, but rather assumes it. Thus, relevant evidence that the FCRI captures a single construct is lacking.

\section{Variability in FCRI-SF Clinical Cut-Offs}

Studies investigating the screening capacity of the FCRISF have recommended different cut-offs for identifying potential clinical FCR cases. FCRI-SF clinical cut-offs were originally established in a study of mixed FrenchCanadian cancer patients $(n=60)$, comparing FCRI-SF scores with results of a purpose-designed semi-structured interview of FCR administered by clinical psychologists. Receiver operating characteristic (ROC) curve analysis suggested a cut-off score $\geq 13$ (range 0-36) demonstrated good sensitivity ( $88 \%)$ and specificity $(75 \%)$ to screen for clinical FCR when identifying as many likely cases of clinical FCR (ie high sensitivity) was a priority (eg in a service delivery context). ${ }^{3}$ The study validating the English version of the FCRI used a similar method to establish the cut-off score of the FCRI-SF with 40 English-speaking mixed cancer patients. ${ }^{14}$ However, both that study and another of 167 ConquerFear RCT participants found that a cut-off $\geq 22$ may be better suited to identifying those with clinical FCR, particularly when greater specificity is needed (eg, eligibility screening for a high-intensity FCR treatment trial). ${ }^{51}$

Variability in FCRI clinical cut-offs may reflect problematic FCRI-SF items making a disproportionate contribution to a positive screen for clinical FCR. For instance, long-term cancer survivors may be more likely to score 4 (several years) on the item "How long have you been thinking about the possibility of cancer recurrence?", which is almost one third of the original 13/36 cut-off. Advanced cancer survivors may also be more likely to be identified as probable clinical FCR cases due to answering FCRI-SF items in a way that reflects their increased likelihood of recurrence/progression and rationality of associated worries, even if such thoughts are not causing significant distress or functional impairment.

\section{Does the FCRI-SF Map on to Key Characteristics of Clinical FCR?}

Part of the reason for the lack of specificity (ie, high false positive rate) of the FCRI-SF in detecting likely cases of clinical FCR may be that many items do not assess features clinicians and researchers consider to be characteristic of clinical FCR. In a recent Delphi study involving 65 international FCR experts, four key characteristics of clinical FCR were identified: (1) high levels of preoccupation; (2) high levels of worry; (3) that are persistent; and (4) hypervigilance to bodily symptoms. ${ }^{52}$ The FCRI-SF arguably assess levels of preoccupation and worry, however, the item assessing duration/persistence asks how long respondents have been "thinking about the possibility of cancer recurrence". This may lead survivors many years 
post-diagnosis who have occasional, rather than persistent, thoughts (not necessarily worries) about recurrence, to score highly on this item, despite having inconsistent and low levels of worry causing little preoccupation. The FCRI-SF also includes two items related to estimations of likelihood of cure and risk of recurrence, which may cause respondents with an inflated perception of their recurrence risk (but not a high level of related worry), or who have come to terms with a poorer prognosis, to screen positively for clinical FCR despite limited worry or preoccupation. In addition to assessing features not presently considered characteristic of clinical FCR, the FCRI-SF does not assess hypervigilance to bodily symptoms.

\section{Does the FCRI Adequately Address Fear of Progression?}

Fear of progression (FoP) is a common experience for people with chronic illness. ${ }^{53,54}$ The current FCR definition $^{1}$ refers not only to fear of recurrence experienced by cancer survivors with no evidence of disease but also to FoP experienced by patients with active cancer. One of the arguments for this broad definition has been that the distinction between "patients" and "survivors" is far from clear-cut, not only in the clinical oncology setting, but also in how people diagnosed with cancer view themselves. Patients may differ from the oncology staff with respect to how they view the intention of their treatment. Some patients receiving adjuvant treatment may, for example, consider themselves cured, while others do not. Thus, even when two patients have identical objective clinical status, one may fear "cancer progression" while the other fears "cancer recurrence". On the other hand, it may still make sense to distinguish between cancer patients and survivors, depending on their treatment status. Individuals in adjuvant treatment will often be considered survivors, as they are no longer being treated for active cancer in contrast to patients in treatment with curative intent or in life-sustaining or palliative treatment. Furthermore, most disease-free survivors are likely to experience their cancer-related fears as FCR, while patients undergoing treatment for active cancer would commonly describe their fears as FoP.

Given the contextual challenges in assessing cancerrelated fears outlined above, the question remains if FCR and FoP are conceptually similar and can be assessed with the same instrument, or whether they should be differentiated and assessed with instruments designed specifically for each circumstance. In alignment with the current FCR definition, the FCRI was developed to assess both FCR and FoP with the exact same items. However, certain FCRI items may not adequately address FoP and result in different scores. For example, the item, "I believe that I am cured and that the cancer will not come back", may not be meaningful to cancer patients with active cancer who do not experience themselves as cured, even if they think progression of their cancer is unlikely. Further clarification is needed regarding the extent to which FCR and FoP are alike, and whether the FCRI can be used to assess both concepts.

\section{Potential Development of an Alternative FCRI Short Form}

Short-form instruments are typically designed to be broadly representative of the complete instrument from which they are derived, whereas the FCRI-SF is simply one of the long-form's seven subscales. This prioritisation of severity over the other six domains is reasonable, as the severity subscale most directly reflects FCR itself, rather than its correlates. ${ }^{49}$ An alternative approach more aligned with the common view of what a short form would be to select a subset of the most appropriate items from the full set of 42. In selecting an approach to short-form development, the purpose of the short form should be considered. For example, if the purpose is simply to reduce participant burden, then the FCRI-SF achieves this but at the expense of content validity, ie, the short instrument does not capture the same breadth of content as the full instrument. If, however, the objective is to develop a short instrument for screening purposes, then the FCRI-SF is appropriate, provided that the level of fear is what matters most for screening an individual as a clinical case. As described above, current work on screening ${ }^{52}$ does not align with this view, focusing not only on fear but some of its consequences (eg, functional impairment).

\section{Alternative FCR Measures}

Although the FCRI is the instrument most commonly used to capture FCR, other instruments have been developed for this purpose. Thewes et $\mathrm{a}^{10}$ provide a thorough review of those developed up to 2012 and found that the FCRI and the Fear of Progression Questionnaire had the strongest psychometric properties of the longer measures (4.5/7 Medical Outcomes Trust criteria), although the latter examines fear of progression rather than recurrence. The 
5-item Assessment of Survivor Concerns Scale ${ }^{55}$ was the best scoring $(2.5 / 7)$ brief measure.

Instruments developed subsequently have tended to comprise fewer items than earlier instruments. Thewes et $\mathrm{al}^{56}$ developed the Concerns About Recurrence Questionnaire, a four-item unidimensional instrument, mostly adapted from existing instruments, and found evidence for its validity and reliability. Humphris et $\mathrm{al}^{57}$ developed two unidimensional instruments - the FCR4 and FCR7 - with four and seven items, respectively. More recently, with growing emphasis on developing short screening tools, Rudy et $\mathrm{al}^{58}$ provided some initial data supporting the use of a single item.

\section{Implications for Clinical Practice}

Despite the limitations of the FCRI, it remains one of the most useful tools for clinicians given a) its good psychometric properties; b) its availability in multiple languages and use with multiple age groups, cancer types, and some minority groups, a testimony to its generalizability; c); the existence of clinical cut-off scores and enough accumulated research, such as the results of the meta-analyses presented in this paper, to situate patients according to some sort of "norm". For the latter point, which is crucial when evaluating FCR treatment efficacy, we have to consider the debate about the appropriate cut-off score to use and that the FCRI-SF appears to lack specificity, ie may over identify patients as having clinical FCR. This lack of specificity means that the FCRI-SF may not adequately capture clinically significant improvements after treatment completion, as evidenced by a high proportion of patients reporting FCRI-SF scores $>13$ post-intervention, despite significant improvements in their FCRI-total score. Presently, following identification of potential clinical FCR using the FCRI-SF, clinicians are encouraged to discuss the perceived severity and impact the fear has on patients' lives and to use the four criteria for clinical FCR that were proposed by Mutsaers et al to confirm the presence of a clinical issue.

\section{Conclusion}

Fear of cancer recurrence research has exploded in the past two decades but has been hampered to some degree by usage of miscellaneous FCR measures of varying quality. The FCRI has emerged as the most commonly utilised measure. Therefore, this state-of-the-art review provides a critical summary of the FCRI: psychometric properties, results regarding FCR prevalence, severity and correlates; and FCRI limitations and potential improvements, to guide future FCR research and clinical practice.

The FCRI has demonstrated a reasonably robust 7-factor structure across evaluations in multiple languages, although potential for improvements remain. In particular, the Coping and Reassurance subscales have demonstrated less-than-adequate reliability. Further work is also needed establishing the cross-cultural equivalence of the numerous FCRI translations.

Our meta-analysis of FCRI-SF scores revealed a combined weighted mean score of 15.7, which is above the lowest proposed cut-off score $(\geq 13)$ for clinical FCR. Depending on the FCRI-SF cut-off used, between $30.0 \%$ and $53.9 \%$ of the cancer population (ie patients and survivors) seemingly experience clinical FCR. However, most data came from intervention studies samples that may not represent the broader cancer survivor population, due to specific inclusion criteria (eg diagnosis of early breast cancer) or attracting a particular type of participants (eg approach versus avoidant copers). Higher FCRI scores were associated with younger age and female gender, pain/physical symptoms and greater anxiety and depression, as per the broader FCR literature. Associations with clinical factors such as cancer type and time since diagnosis were mixed.

Issues regarding the application and interpretation of the FCRI remain, for instance it is unclear whether the FCRI is well suited to assessing FoP as well as FCR and the meaningfulness of the FCRI total score is debatable. The use of the FCRI-SF to screen for potential cases of clinical FCR is problematic, as items do not clearly map on to recently established characteristics of clinical FCR. Together with FCRI-SF items that may be more sensitive to respondents' cancer stage or time since treatment than their FCR severity, this may partially explain the variable FCRI-SF cut-offs for clinical FCR reported. Development of a short form of the FCRI that is more representative of the broader scale and characteristics of clinical FCR may overcome some of these limitations and help capture the experience of FCR while reducing respondent burden. For screening purposes, alternative measures, such as the FCR-4/7, the CARQ-4 or a single item that can easily be incorporated into broader screening assessments could be considered. While the FCRI has been critical to advancing FCR research to this point, refinements are needed if it is to remain a key FCR assessment tool in future research and clinical practice. 


\section{Acknowledgments}

Dr Smith is funded through a Cancer Institute NSW grant. Please note, authors after the corresponding author are listed in alphabetical order due to the roughly equivalent contributions of all the authors.

\section{Disclosure}

The authors report no conflicts of interest in this work.

\section{References}

1. Lebel S, Ozakinci G, Humphris G, et al. From normal response to clinical problem: definition and clinical features of fear of cancer recurrence. Support Care Cancer. 2016;24(8):3265-3268. doi:10.1007/s00520-016-3272-5

2. Simard S, Thewes B, Humphris G, et al. Fear of cancer recurrence in adult cancer survivors: a systematic review of quantitative studies. J Cancer Surviv. 2013;7(3):300-322. doi:10.1007/s11764-013-0272-z

3. Simard S, Savard J. Screening and comorbidity of clinical levels of fear of cancer recurrence. J Cancer Surviv. 2015;9(3):481-491. doi:10.1007/s11764-015-0424-4

4. Koch L, Jansen L, Brenner H, Arndt V. Fear of recurrence and disease progression in long-term ( $\geq 5$ years) cancer survivors a systematic review of quantitative studies. Psychooncology. 2013;22(1):1-11. doi:10.1002/pon.3022

5. Crist JV, Grunfeld EA. Factors reported to influence fear of recurrence in cancer patients: a systematic review. Psychooncology. 2013;22(5):978-986. doi:10.1002/pon.3114

6. Lebel S, Tomei C, Feldstain A, Beattie S, McCallum M. Does fear of cancer recurrence predict cancer survivors' health care use? Support Care Cancer. 2013;21(3):901-906. doi:10.1007/s00520-012-1685-3

7. Thewes B, Butow P, Bell ML, et al. Fear of cancer recurrence in young women with a history of early-stage breast cancer: a cross-sectional study of prevalence and association with health behaviours. Support Care Cancer. 2012;20(11):2651-2659. doi:10.1007/s00520-011-1371-x

8. Champagne A, Ivers H, Savard J. Utilization of health care services in cancer patients with elevated fear of cancer recurrence. Psychooncology. 2018;27(8):1958-1964. doi:10.1002/pon.4748

9. Tauber NM, O’Toole MS, Dinkel A, et al. Effect of psychological intervention on fear of cancer recurrence: a systematic review and meta-analysis. J Clin Oncol. 2019;37(31):2899-2915. doi:10.1200/ JCO.19.00572

10. Thewes B, Butow P, Zachariae R, Christensen S, Simard S, Gotay C. Fear of cancer recurrence: a systematic literature review of self-report measures. Psychooncology. 2012;21(6):571-587. doi:10.1002/ pon. 2070

11. Simard S, Savard J. Fear of Cancer Recurrence Inventory: development and initial validation of a multidimensional measure of fear of cancer recurrence. Support Care Cancer. 2009;17(3):241-251. doi:10.1007/s00520-008-0444-y

12. Vickberg SMJ. The concerns about recurrence scale (CARS): a systematic measure of women's fears about the possibility of breast cancer recurrence. Ann Behav Med. 2003;25(1):16-24.

13. Simard S, Savard J, Ivers H. Fear of cancer recurrence: specific profiles and nature of intrusive thoughts. J Cancer Surviv. 2010;4 (4):361-371. doi:10.1007/s11764-010-0136-8

14. Lebel S, Simard S, Harris C, et al. Empirical validation of the English version of the Fear of Cancer Recurrence Inventory. Qual Life Res. 2016;25(2):311-321. doi:10.1007/s11136-015-1088-2

15. Grant MJ, Booth A. A typology of reviews: an analysis of 14 review types and associated methodologies. Health Inf Libr J. 2009;26 (2):91-108. doi:10.1111/j.1471-1842.2009.00848.x
16. Lin C-R, Chen S-C, Simard S, Chang JT-C, Lai Y-H. Psychometric testing of the Fear of Cancer Recurrence Inventory-caregiver Chinese version in cancer family caregivers in Taiwan. Psychooncology. 2018;27(6):1580-1588. doi:10.1002/pon.4697

17. Hovdenak Jakobsen I, Jeppesen MM, Simard S, Thaysen HV, Laurberg S, Juul T. Initial validation of the Danish version of the Fear of Cancer Recurrence Inventory (FCRI) in colorectal cancer patients. J Cancer Surviv. 2018;12(6):723-732. doi:10.1007/s11764018-0709-5

18. van Helmondt SJ, van der Lee ML, de Vries J. Translation and validation of the Dutch version of the Fear of Cancer Recurrence Inventory (FCRI-NL). $J$ Psychosom Res. 2017;102:21-28. doi:10.1016/j.jpsychores.2017.09.001

19. Shin J, Goo A, Ko H, et al. Validation study for the Korean version of fear of cancer recurrence inventory. J Korean Med Sci. 2017;32 (11):1792-1799. doi:10.3346/jkms.2017.32.11.1792

20. Liu J, Mahendran R, Chua SM, et al. Validation of the English and Mandarin versions of the Fear of Cancer Recurrence Inventory in an Asian population. $J$ Health Psychol. 2017;25(5):617-628. doi:10.1177/1359105317727819

21. Bateni FS, Rahmatian M, Kaviani A, Simard S, Soleimani M, Nejatisafa -A-A. The Persian Version of the Fear of Cancer Recurrence Inventory (FCRI): translation and evaluation of its psychometric properties. Arch Breast Cancer. 6(4):174-180. doi:10.19187/abc. 201964174-180

22. Eyrenci A, Sertel Berk HÖ. Validity and reliability of the Turkish version of fear of cancer recurrence inventory. Turk Onkol Derg. 2018;33(2):54-64. doi:10.5505/tjo.2018.1759

23. van Helmondt SJ, van der Lee ML, Bisseling EM, Lodder P, de Vries J. Factor structure of the Fear of Cancer Recurrence Inventory (FCRI): comparison of international FCRI factor structure data and factor analysis of the Dutch FCRI-NL using three predominantly breast cancer samples. Eur J Cancer Care (Engl). 2020.

24. Galica J, Brennenstuhl S, Maheu C, Townsley C, Metcalfe K. Examining the dimensionality of the Fear of Cancer Recurrence Inventory. Psychooncology. 2018;27(11):2602-2608. doi:10.1002/ pon. 4839

25. Ponterotto JG, Charter RA. Statistical extensions of ponterotto and Ruckdeschel's (2007) reliability matrix for estimating the adequacy of internal consistency coefficients. Percept Mot Skills. 2009;108 (3):878-886. doi:10.2466/PMS.108.3.878-886

26. Custers JAE, Gielissen MFM, de Wilt JHW, et al. Towards an evidence-based model of fear of cancer recurrence for breast cancer survivors. J Cancer Surviv. 2017;11(1):41-47. doi:10.1007/s11764016-0558-z

27. Costa DSJ, Dieng M, Cust AE, Butow PN, Kasparian NA. Psychometric properties of the Fear of Cancer Recurrence Inventory: an item response theory approach. Psychooncology. 2016;25(7):832-838. doi:10.1002/pon.4018

28. Frost MH, Reeve BB, Liepa AM, Stauffer JW, Hays RD. What is sufficient evidence for the reliability and validity of patient-reported outcome measures? Value Health. 2007;10:S94-S105. doi:10.1111/ j.1524-4733.2007.00272.x

29. Custers JAE, Gielissen MFM, Janssen SHV, de Wilt JHW, Prins JB. Fear of cancer recurrence in colorectal cancer survivors. Support Care Cancer. 2016;24(2):555-562. doi:10.1007/s00520-015-2808-4

30. Smith AB, Sharpe L, Thewes B, et al. Medical, demographic and psychological correlates of fear of cancer recurrence (FCR) morbidity in breast, colorectal and melanoma cancer survivors with probable clinically significant FCR seeking psychological treatment through the ConquerFear study. Support Care Cancer. 2018;26 (12):4207-4216. doi:10.1007/s00520-018-4294-y

31. Savard J, Ivers $H$. The evolution of fear of cancer recurrence during the cancer care trajectory and its relationship with cancer characteristics. J Psychosom Res. 2013;74(4):354-360. doi:10.1016/ j.jpsychores.2012.12.013 
32. Van Liew JR, Christensen AJ, Howren MB, Karnell LH, Funk GF Fear of recurrence impacts health-related quality of life and continued tobacco use in head and neck cancer survivors. Health Psychol. 2014;33(4):373-381. doi:10.1037/a0032680

33. Mahendran R, Liu J, Kuparasundram S, et al. Fear of cancer recurrence among cancer survivors in Singapore. Singapore Med J. 2020. doi:10.11622/smedj.2020007

34. Savard J, Savard M-H, Caplette-Gingras A, Casault L, Camateros C. Development and feasibility of a group cognitive-behavioral therapy for fear of cancer recurrence. Cogn Behav Pract. 2018;25 (2):275-285. doi:10.1016/j.cbpra.2017.08.001

35. Butow P, Kelly S, Thewes B, Hruby G, Sharpe L, Beith J. Attentional bias and metacognitions in cancer survivors with high fear of cancer recurrence. Psychooncology. 2015;24(4):416-423. doi:10.1002/pon.3659

36. Ng DWL, Kwong A, Suen D, et al. Fear of cancer recurrence among Chinese cancer survivors: prevalence and associations with metacognition and neuroticism. Psychooncology. 2019;28(6):1243-1251. doi: $10.1002 /$ pon. 5073

37. Lane BE, Garland SN, Chalifour K, et al. Prevalence and factors associated with fear of recurrence in a mixed sample of young adults with cancer. J Cancer Surviv. 2019;13(6):842-851. doi:10.1007/ s11764-019-00802-9

38. Hovdenak Jakobsen I, Juul T, Thaysen HV, Johansen C, Laurberg S. On behalf of the $\mathrm{F}$ project group. Differences in baseline characteristics and 1-year psychological factors between participants and non-participants in the randomized, controlled trial regarding patient-led follow-up after rectal cancer (FURCA). Acta Oncol. 2019;58(5):627-633. doi:10.1080/0284186X.2019.1581948

39. Wijayanti T, Afiyanti Y, Rahmah H, Milanti A. Fear of cancer recurrence and social support among Indonesian gynecological cancer survivors. Arch Oncol. 2018;24(2):12-19. doi:10.2298/AOO180201004W

40. Jeppesen MM, Jensen PT, Hansen DG, Christensen RD, Mogensen O. Patient-initiated follow up affects fear of recurrence and healthcare use: a randomised trial in early-stage endometrial cancer. BJOG Int J Obstet Gynaecol. 2018;125(13):1705-1714. doi:10.1111/1471-0528.15396

41. Dirkse D, Hadjistavropoulos HD, Alberts NA, et al. Making Internet-delivered cognitive behaviour therapy scalable for cancer survivors: a randomized non-inferiority trial of self-guided and technician-guided therapy. J Cancer Surviv. 2019. doi:10.1007/ s11764-019-00810-9

42. Ng DWL, Foo -C-C, Ng SSM, et al. The role of metacognition and its indirect effect through cognitive attentional syndrome on fear of cancer recurrence trajectories: a longitudinal study. Psychooncology. 2020;29(2):271-279. doi:10.1002/pon.5234

43. Thewes B, Bell ML, Butow P. Fear of cancer recurrence in young early-stage breast cancer survivors: the role of metacognitive style and disease-related factors. Psychooncology. 2013;22(9):2059-2063. doi: $10.1002 /$ pon. 3252

44. Galica J, Maheu C, Brennenstuhl S, Townsley C, Metcalfe K. Examining predictors of Fear of Cancer Recurrence Using Leventhal's Commonsense Model: distinct implications for oncology nurses. Cancer Nurs. 2019. doi:10.1097/NCC.0000000000000760

45. Kelada L, Wakefield CE, Heathcote LC, et al. Perceived cancer-related pain and fatigue, information needs, and fear of cancer recurrence among adult survivors of childhood cancer. Patient Educ Couns. 2019;102(12):2270-2278. doi:10.1016/j.pec.2019.06.022

46. Lopez C, Charles C, Rouby P, et al. Relations between arthralgia and fear of recurrence: results of a cross-sectional study of breast cancer patients treated with adjuvant aromatase inhibitors therapy. Support Care Cancer. 2015;23(12):3581-3588. doi:10.1007/s00520-015-2722-9

47. Liu J, Peh C-X, Simard S, Griva K, Mahendran R. Beyond the fear that lingers: the interaction between fear of cancer recurrence and rumination in relation to depression and anxiety symptoms. J Psychosom Res. 2018;111:120-126. doi:10.1016/j.jpsychores.2018.06.004
48. Walburg V, Rueter M, Lamy S, et al. Fear of cancer recurrence in Non- and Hodgkin lymphoma survivors during their first three years of survivorship among French patients. Psychol Health Med. 2019;24 (7):781-787. doi:10.1080/13548506.2019.1574354

49. Costa DSJ, Smith AB, Fardell JE. The sum of all fears: conceptual challenges with measuring fear of cancer recurrence. Support Care Cancer. 2016;24(1):1-3. doi:10.1007/s00520-015-2943-y

50. Costa DSJ. Screening for clinical levels of fear of cancer recurrence. Psychooncology. 2017;26(11):2002-2003. doi:10.1002/pon.4390

51. Fardell JE, Jones G, Smith AB, et al. Exploring the screening capacity of the Fear of Cancer Recurrence Inventory-Short Form for clinical levels of fear of cancer recurrence. Psychooncology. 2018;27(2):492-499. doi:10.1002/pon.4516

52. Mutsaers B, Butow P, Dinkel A, et al. Identifying the key characteristics of clinical fear of cancer recurrence: an international Delphi study. Psychooncology. 2020;29(2):430-436. doi:10.1002/pon.5283

53. Herschbach P, Berg P, Dankert A, et al. Fear of progression in chronic diseases: psychometric properties of the Fear of Progression Questionnaire. J Psychosom Res. 2005;58(6):505-511. doi:10.1016/ j.jpsychores.2005.02.007

54. Lebel S, Mutsaers B, Tomei C, et al. Health anxiety and illness-related fears across diverse chronic illnesses: a systematic review on conceptualization, measurement, prevalence, course, and correlates. PLoS One. 2020;15(7):e0234124. doi:10.1371/journal. pone. 0234124

55. Gotay CC, Pagano IS. Assessment of Survivor Concerns (ASC): a newly proposed brief questionnaire. Health Qual Life Outcomes. 2007;5(1):15. doi:10.1186/1477-7525-5-15

56. Thewes B, Zachariae R, Christensen S, Nielsen T, Butow P. The Concerns About Recurrence Questionnaire: validation of a brief measure of fear of cancer recurrence amongst Danish and Australian breast cancer survivors. $J$ Cancer Surviv. 2015;9(1):68-79. doi:10.1007/s11764-014-0383-1

57. Humphris GM, Watson E, Sharpe M, Ozakinci G. Unidimensional scales for fears of cancer recurrence and their psychometric properties: the FCR4 and FCR7. Health Qual Life Outcomes. 2018;16 (1):30. doi:10.1186/s12955-018-0850-x

58. Rudy L, Maheu C, Körner A, Lebel S, Gélinas C. The FCR-1: initial validation of a single-item measure of fear of cancer recurrence. Psychooncology. n/a(n/a). doi:10.1002/pon.5350

59. Dieng M, Butow PN, Costa DSJ, et al. Psychoeducational intervention to reduce fear of cancer recurrence in people at high risk of developing another primary melanoma: results of a randomized controlled trial. J Clin Oncol. 2016;34(36):4405-4414. doi:10.1200/JCO.2016.68.2278

60. Russell L, Ugalde A, Orellana L, et al. A pilot randomised controlled trial of an online mindfulness-based program for people diagnosed with melanoma. Support Care Cancer. 2019;27(7):2735-2746. doi:10.1007/s00520-018-4574-6

61. Bell KJL, Mehta Y, Turner RM, et al. Fear of new or recurrent melanoma after treatment for localised melanoma. Psychooncology. 2017;26(11):1784-1791. doi:10.1002/pon.4366

62. Merckaert I, Lewis F, Delevallez F, et al. Improving anxiety regulation in patients with breast cancer at the beginning of the survivorship period: a randomized clinical trial comparing the benefits of single-component and multiple-component group interventions. Psychooncology. 2017;26(8):1147-1154. doi:10.1002/pon.4294

63. van Helmondt SJ, van der Lee ML, van Woezik RAM, Lodder P, de Vries J. No effect of CBT-based online self-help training to reduce fear of cancer recurrence: first results of the CAREST multicenter randomized controlled trial. Psychooncology. 2020;29(1):86-97. doi:10.1002/pon.5233

64. Tesson S, Richards I, Porter D, et al. Women's preferences for contralateral prophylactic mastectomy following unilateral breast cancer: what risk-reduction makes it worthwhile? Breast. 2017;31:233-240. doi:10.1016/j.breast.2016.11.025 
65. Peng L, Huang W, Zhang W, et al. Psychometric properties of the short form of the Fear of Cancer Recurrence Inventory (FCRI) in Chinese Breast Cancer Survivors. Front Psychiatry. 2019:10. doi:10.3389/fpsyt.2019.00537.

66. Zdenkowski N, Butow P, Spillane A, et al. Patient-reported outcomes with neoadjuvant vs adjuvant systemic therapy for operable breast cancer. Breast. 2019;46:25-31. doi:10.1016/j. breast.2019.04.003

67. Corter AL, Broom R, Porter D, Harvey V, Findlay M. Predicting nonadherence to adjuvant endocrine therapy in women with early stage breast cancer. Psychooncology. 2018;27(9):2096-2103. doi:10.1002/pon.4771

68. Guimond A-J, Ivers H, Savard J. Is emotion regulation associated with cancer-related psychological symptoms? Psychol Health. 2019;34(1):44-63. doi:10.1080/08870446.2018.1514462

69. Zdenkowski N, Butow P, Spillane A, et al. Single-arm longitudinal study to evaluate a decision aid for women offered Neoadjuvant systemic therapy for operable breast cancer. JNCCN J Natl Compr Cancer Netw. 2018;16(4):378-385. doi:10.6004/jnccn.2017.7063

70. Petzel MQB, Parker NH, Valentine AD, et al. Fear of cancer recurrence after curative pancreatectomy: a cross-sectional study in survivors of pancreatic and periampullary tumors. Ann Surg Oncol. 2012;19(13):4078-4084. doi:10.1245/s10434-012-2566-1

71. van de Wal M, van Oort I, Schouten J, Thewes B, Gielissen M, Prins J. Fear of cancer recurrence in prostate cancer survivors. Acta Oncol. 2016;55(7):821-827. doi:10.3109/0284186X.2016.1150607

72. Nelson AM, Jim HSL, Small BJ, et al. Sleep disruption among cancer patients following autologous hematopoietic cell transplantation. Bone Marrow Transplant. 2018;53(3):307-314. doi:10.1038/s41409017-0022-3

73. Hong SJ, Shin N-M, Jung S. A predictive model of fear of cancer recurrence for patients undergoing chemotherapy. Support Care Cancer. 2020;28(9):4173-4181. doi:10.1007/s00520-019-05245-7
74. Compen F, Bisseling E, Schellekens M, et al. Face-to-face and internet-based mindfulness-based cognitive therapy compared with treatment as usual in reducing psychological distress in patients with cancer: a multicenter randomized controlled trial. J Clin Oncol. 2018;36(23):2413-2421. doi:10.1200/JCO.2017.76.5669

75. Butow PN, Turner J, Gilchrist J, et al. Randomized trial of ConquerFear: a novel, theoretically based psychosocial intervention for fear of cancer recurrence. J Clin Oncol. 2017;35(36):4066-4077. doi:10.1200/JCO.2017.73.1257

76. Van De Wal M, Thewes B, Gielissen M, Speckens A, Prins J. Efficacy of blended cognitive behavior therapy for high fear of recurrence in breast, prostate, and colorectal cancer survivors: the SWORD study, a randomized controlled trial. J Clin Oncol. 2017;35 (19):2173-2183. doi:10.1200/JCO.2016.70.5301

77. Mahendran R, Liu J, Kuparasundram S, Griva K. Validation of the English and simplified Mandarin versions of the Fear of Progression Questionnaire-Short Form in Chinese cancer survivors. BMC Psychol. 2020;8:1. doi:10.1186/s40359-020-0374-0

78. Shin J, Shin DW, Lee J, et al. Association between perception of care coordination and health outcomes in Korean cancer survivors. Health Qual Life Outcomes. 2020;18(1):21. doi:10.1186/s12955-020-1279-6

79. Fisher PL, Byrne A, Fairburn L, Ullmer H, Abbey G, Salmon P. Brief metacognitive therapy for emotional distress in adult cancer survivors. Front Psychol. 2019;10(JAN). doi:10.3389/ fpsyg.2019.00162

80. Murphy MJ, Newby JM, Butow P, et al. Randomised controlled trial of internet-delivered cognitive behaviour therapy for clinical depression and/or anxiety in cancer survivors (iCanADAPT Early). Psychooncology. 2020;29(1):76-85. doi:10.1002/pon.5267

81. Lebel S, Maheu C, Tomei C, et al. Towards the validation of a new, blended theoretical model of fear of cancer recurrence. Psychooncology. 2018;27(11):2594-2601. doi:10.1002/pon.4880
Psychology Research and Behavior Management

\section{Publish your work in this journal}

Psychology Research and Behavior Management is an international, peer-reviewed, open access journal focusing on the science of psychology and its application in behavior management to develop improved outcomes in the clinical, educational, sports and business arenas. Specific topics covered in the journal include: Neuroscience, memory and decision making; Behavior modification and management; Clinical applications; Business and sports performance management; Social and developmental studies; Animal studies. The manuscript management system is completely online and includes a very quick and fair peer-review system, which is all easy to use. Visit http://www. dovepress.com/testimonials.php to read real quotes from published authors. 